\title{
Factors associated with delay in seeking treatment among women with pelvic organ prolapse at selected general and referral hospitals of Southern Ethiopia, 2020
}

\author{
Asfaw Borsamo ${ }^{1}$, Mohammed Oumer $^{2,3^{*}}$, Yared Asmare² and Ayanaw Worku²
}

\begin{abstract}
Background: Pelvic organ prolapse (POP) is the descent of the vaginal wall, cervix, uterus, bladder, and rectum downward into the vaginal canal. Its prevalence is higher among women in developing countries because women are more prone to risk factors. In Ethiopia, women with prolapse seek treatments at advanced stages of prolapse; hence, surgical management has been widely practicing. Therefore, it was found to be very important to conduct research that assesses factors hindering early treatments in Southern Ethiopia. This study aimed to find out factors associated with the delay in seeking treatment of pelvic organ prolapse among patients at selected general and referral hospitals of Southern Ethiopia.
\end{abstract}

Methods: Cross-sectional study design was employed in 123 participants of seven randomly selected General and Referral Hospitals of Southern Ethiopia from February 01 to April 30, 2020, by using a structured questionnaire. Pretrained two midwives in each center were deployed to collect data. Physicians performed diagnosis and physical examination. Data were entered and coded using EPI INFO version 7 and exported into SPSS version 25 for analysis. Bivariate and multivariable logistic regression analyses were performed. The goodness of fit was assessed by using the Hosmer and Lemeshow goodness test.

Results: In this study, out of 123 clinically diagnosed POP cases, nearly half of them were stage III, and over one-third were stage IV. Therefore, $84.6 \%$ (104 participants) of the respondents were delayed for the treatment of POP. The mean length of delay for POP treatments was $36.41 \pm 3.95$ months. After adjusting for covariates, lack of supports [AOR (Adjusted Odds Ratio) $=5.2(95 \% \mathrm{Cl} 1.4-19.5)]$, low-income $[A O R=5.8(95 \% \mathrm{Cl} 1.1-19.66)]$, and fear of social stigma $[A O R=4.7(95 \% \mathrm{Cl} 1.2-18.59)]$ were significant factors for delayed treatments.

Conclusions: Most of the POP patients were delayed for POP treatments. Factors like lack of support, low-income, and fear of losing social value/stigma were associated with treatment delay. Screening for the POP cases, educating (making awareness) the community about this devastating disease to facilitate early treatment and to avoid social stigma, and raising access to treatment by making the nearby hospitals equipped with facilities to treat POP are recommended.

Keywords: Pelvic organ prolapse, Delay in seeking treatment, Ethiopia

*Correspondence: mohammedoumer58@gmail.com

3 Department of Epidemiology, Institute of Public Health, College

of Medicine and Health Sciences, University of Gondar, Gondar, Ethiopia

Full list of author information is available at the end of the article

\section{Background}

Pelvic organ prolapse (POP) is the herniation of the uterus, the cervix, the vaginal wall, the bladder, and the rectum downward along the vaginal lumen [1]. It is a 
common gynecological problem, which is more prevalent in developing countries, that affect the life quality of women by limiting emotional, physical, psychosocial, and sexual functions $[2,3]$.

The prevalence of POP varies widely from population to population depending on the level of exposure to the risk, level of awareness about POP, and level of the socioeconomic status. Universally, the prevalence of this problem is about $3.5 \%$ to $15 \%$ when it is studied by defining symptoms and it reaches up to $64.6 \%$ when studied based on objective findings, which are directly measured by the experts [3-8].

Women in the developed countries who have access to modern health care can benefit from the advances that have been made in treating POP. Moreover, women in developed nations have an awareness of the problem, high literacy rate, and high-income level with excellent health facilities that can screen the POP very early. Delaying for treatment is also affected by high parity, little or no access to health care, and the presence of problems associated with POP [9].

The prevalence of POP in Africa varies widely from country to country: 64.6\% in Tanzania [5], 19.4\% in Egypt [10], and $6.5 \%$ in Nigeria [11]. The prevalence of the POP in Ethiopia is also highly variable ranging from 1 to $56.4 \%$ $[3,7,12]$. For instance, $40.7 \%$ among gynecologic case in Jimma Hospitals [13], 15\% among gynecologic case in Saint Paulos Hospital, Addis Abeba [14], 56.4\% in Dabat community, Gondar [3], 20.9\% in Kersa community, Ethiopia [7], and 13.3\% in Benchi Maji Zone [15].

Essentially, delaying the POP treatments worsens the problem further in Ethiopia. Many women are silent victims of uterovaginal prolapse, hiding the problem owing to fearing social stigma. Despite uterovaginal prolapse is a matter of discomfort for women, it affects many aspects of their daily life $[12,16]$. According to a community-based objectively measured study at Dabat district, Gondar, the prevalence of POP is about $56.4 \%$, however, only patients with advanced stages of POP (3.2\%) visited the hospitals, even if free service is providing [3].

According to few studies assessing reasons for delayed treatments among women within Ethiopia, the possible reasons are lack of support, low awareness, low literacy, unavailability of the treatment center, low income, and disclosure of the problem or fear of social stigma $[12,16]$. In Ethiopia, although the non-governmental organizations in collaboration with the Federal Ministry of Health tried to deliver free services for the victims integrating with obstetric fistula care, the women kept home hiding their problems even from their close partners. This indicates that little attention is given to health education, and creating awareness on the problem. Besides, few studies were conducted on reasons hindering early treatment $[12,16]$.

There is a scarcity of the study assessing factors associated with the delay in seeking treatment among women with POP in Southern Ethiopia. Notably, exposing factors that hinder early treatments of POP initiates up responsible bodies to take interventional actions at the early stages of the problem. Therefore, it was found to be very important to conduct research that assesses factors associated with the delay in seeking treatment among women with POP in South Nations Nationalities and Peoples' Region (SNNPR).

\section{Methods}

\section{Study design and setting}

The hospital-based cross-sectional study design was conducted from February 01 to April 30, 2020, in seven (out of thirteen hospitals) randomly selected governmental general and referral hospitals of southern Ethiopia. All gynecologic patients diagnosed with a POP at selected governmental general and referral hospitals of Southern Ethiopia were included in the study. A total of 123 eligible POP women participated in the study.

\section{Measurement}

POP was evaluated and described using a standardized Pelvic Organ Prolapse Quantitative Examination Tool. In this technique, the hymen ring (remnant) is considered as a reference point $[1,17]$. The stages of POP were presented in Table 1.

Table 1 The pelvic organ prolapse staging, 2020

\begin{tabular}{ll}
\hline Stages & Description \\
\hline 0 & No prolapse $[1,17]$ \\
I & The leading-edge of the prolapse is $1 \mathrm{~cm}$ above the level of the hymen $(>-1 \mathrm{~cm})[1,17]$ \\
III & The leading-edge of the prolapse lies through the plane of hymen between $-1 \mathrm{~cm}$ and $+1 \mathrm{~cm}[1,17]$ \\
IV & The most distal portion of the prolapse is between $+1 \mathrm{~cm}$ and $+2 \mathrm{~cm}$ below hymen $[1,17]$ \\
\hline
\end{tabular}




\section{Operational definitions}

Delayed treatment of POP: Advanced stage prolapses treated with surgical management regardless of the duration of the onset of symptoms [1]. Early treatment of POP: Early-stage prolapses treated with conservative management regardless of the duration of the onset of symptoms [1]. Low income: Those who earn less than 1200 Birr monthly were considered as low income and, practically, those women who have the interest to go hospital but delay in seeking treatment due to the lack of money [12, 18]. Lack of support: Those women who delayed in seeking treatments because they do not have a person who supports them financially and emotionally $[12,16]$. Culture influence: In some cultures in Ethiopia, it is extremely shameful exposing, discussing, and talking about anything related to genitalia (sensitive body parts), even to their daughters and children [12]. Being busy: Those women who delayed in seeking treatments because they are busy with works and with different responsibilities [19]. Fear of social stigma: A woman with POP who delayed in seeking treatments due to the high concern of social discrimination [11, 12].

\section{Data collection tools, techniques, and procedures}

Data were collected using the interviewer-guided structured questionnaire, having two parts: The first part assessed objective findings, and the second part assessed factors for delayed treatments. To assess objective findings, physicians first took the surgical and medical history of the patients. Then, they performed vaginal examinations and diagnoses. Based on Standardized Pelvic Organ Prolapse Quantitative Examination Tool, physicians determined the stages of POP. After the physician decided on management, participants were interviewed.

The questionnaire was developed by the investigators by reviewing different related literature $[3,11,12,16$, 20]. Then, the questionnaire was evaluated by the experts in the profession to ensure validity. The questionnaire was first prepared in English, and, then, appropriately translated into the Amharic language. Later, translated back to English to ensure the accuracy of the meaning. A pretest was carried out on $10 \%$ of the sample size at the Shashemene General Hospital, Oromia, Ethiopia, and Bushulo General Hospital, Sidama, Ethiopia (private obstetrics and gynecology center) mainly to check the general approachability, validity, and feasibility of the questionnaire. As a result, corrections and modifications were made to the questionnaire accordingly. Two midwives collected the data using the Amharic version questionnaire under strict supervision.

\section{Data quality control and management}

Data quality was controlled through the provision of one-day training to the data collectors about the overall objective of the study, the definition of terms and concepts, approaching of respondents, data collection tools, and techniques of interviewing. The collected data were also cross-checked for its completeness, consistency, accuracy, and clarity daily. The investigators carried out close site supervision during the whole data collection period to monitor overall data collection quality.

\section{Data processing and analysis}

After data collection, each questionnaire was again visually checked for completeness, clarity, and accuracy. Data were coded and entered into EPI INFO version 7 software and exported to SPSS version 25 statistical software for analysis. Descriptive, bivariate, and multivariable logistic regression analyses were performed. Selected variables that have a $P$ value of $\leq 0.2$ at the bivariate logistic regression analysis were included in the multivariable logistic regression to control all possible confounding factors simultaneously. The goodness of fit was assessed by using the Hosmer and Lemeshow goodness test. Generally, a P value $\leq$ of 0.05 was considered statistically significant.

\section{Results}

Socio-demographic characteristics of the participants

Out of 123 participants, the majority (61\%) were aged $\geq 45$ years. Over two-thirds $(67.48 \%)$ of the participants did not attend formal school at all. About $66.70 \%$ of participants were rural residents and $71.54 \%$ were housewives. The majority $(69.01 \%)$ earns $\leq 1200$ Ethiopian birrs monthly (Table 2).

\section{Description of delayed treatments}

One hundred twenty-three cases were clinically diagnosed for POP. Out of 123 POP cases, nearly half (60 participants) of them were stage III, and over one-third (44 participants) were stage IV. Therefore, 84.6\% (104 participants) of the respondents were delayed for the treatment of POP. The mean length of delay for POP treatments was $36.41 \pm 3.95$ months (Table 3 ).

\section{Reasons for delayed treatments}

Out of 123 women with POP, $58.54 \%$ of women complained of a lack of support as the reason for the delayed treatments. The majority $(73.17 \%)$ reasoned out a lack of transportation for delayed treatments (Table 4).

\section{Factors associated with delayed treatments}

On bivariate logistic regression analysis, lack of support, low-income, lack of transportation and road, 
Table 2 Sociodemographic

characteristics of the participants at selected general and referral hospitals of Southern Ethiopia, 2020

\begin{tabular}{|c|c|c|}
\hline Variables & $\begin{array}{l}\text { Frequency } \\
(n=123)\end{array}$ & Percent (\%) \\
\hline \multicolumn{3}{|l|}{ Age } \\
\hline$\leq 35$ & 13 & 10.57 \\
\hline $34-44$ & 35 & 28.46 \\
\hline$\geq 45$ & 75 & 60.97 \\
\hline \multicolumn{3}{|l|}{ Ethnicity } \\
\hline Wolaita & 33 & 26.83 \\
\hline Sidama & 27 & 21.95 \\
\hline Gurage & 16 & 13.00 \\
\hline Hadiya & 14 & 11.38 \\
\hline Amhara & 8 & 6.50 \\
\hline Oromo & 8 & 6.50 \\
\hline Kambata & 7 & 5.70 \\
\hline Gamo & 6 & 4.88 \\
\hline Dorze & 2 & 1.63 \\
\hline Amaro & 1 & 0.81 \\
\hline Halaba & 1 & 0.81 \\
\hline \multicolumn{3}{|l|}{ Religion } \\
\hline Protestant & 68 & 55.28 \\
\hline Orthodox & 43 & 34.96 \\
\hline Muslim & 9 & 7.32 \\
\hline Waka and traditional believers & 7 & 5.69 \\
\hline \multicolumn{3}{|l|}{ Residency } \\
\hline Rural & 82 & 66.70 \\
\hline Urban & 41 & 33.30 \\
\hline \multicolumn{3}{|l|}{ Occupational status } \\
\hline House wife & 88 & 71.54 \\
\hline Farmer & 23 & 18.70 \\
\hline Merchant & 23 & 18.70 \\
\hline Employed & 24 & 19.51 \\
\hline \multicolumn{3}{|l|}{ Educational status } \\
\hline No schooling & 83 & 67.48 \\
\hline Primary & 18 & 14.63 \\
\hline Secondary & 15 & 12.20 \\
\hline Diploma+ & 7 & 5.69 \\
\hline \multicolumn{3}{|l|}{ Marital status } \\
\hline Married & 104 & 84.55 \\
\hline Widowed & 10 & 8.13 \\
\hline Divorced & 9 & 7.32 \\
\hline \multicolumn{3}{|l|}{ Monthly income (ETB) } \\
\hline$>1200$ & 38 & 30.89 \\
\hline$\leq 1200$ & 85 & 69.01 \\
\hline
\end{tabular}

believing POP as normal, fear of losing social value/ stigma, fear of disclosure, maternal age, and educational status were significant reasons for delayed treatments. On multivariable logistic regression analysis,
Table 3 Summary of stages of POP and management types at selected General and Referral Hospitals of Southern Ethiopia, 2020

\begin{tabular}{lcc}
\hline Stage of POP & Frequency & Percentage (\%) \\
\hline Stage I & 0 & 0 \\
Stage II & 19 & 15.4 \\
Stage III & 60 & 48.8 \\
Stage IV & 44 & 35.8 \\
Management types & & \\
Surgically managed & 104 & 84.6 \\
Conservatively managed & 19 & 15.4 \\
\hline
\end{tabular}

lack of support, low-income, and fear of losing social value/stigma were reasons associated with delayed treatments of POP patients. Accordingly, women with a lack of support were five times $[\mathrm{AOR}=5.2(95 \% \mathrm{CI}$ 1.42-19.5)] more likely to delay treatments of POP than their counterparts. Women with low-income were nearly six times $[\mathrm{AOR}=5.8(95 \%$ CI $1.1-19.66)]$ more likely to delay treatments of POP than women with higher income. Women who fear social stigma was about nearly five times $[\mathrm{AOR}=4.7$ (95\% CI 1.2-18.59)] more likely to delay treatments as compared to their counterparts (Table 5). The value of the Hosmer and Lemeshow goodness of fit test was 0.332 .

\section{Discussion}

This study primarily assessed factors associated with the delay in seeking treatment among women with POP at selected general and referral hospitals of Southern Ethiopia. Accordingly, lack of support, low income, and fear of social stigma were significant factors associated with delayed treatments of POP patients. This study also assessed reasons for delayed treatments among POP patients at selected general and referral hospitals of Southern Ethiopia. Out of 123 POP patients, only $15.4 \%$ of POP patients came with earlier stages of POP. The mean length of delay for POP treatments was $36.41 \pm 3.95$ months. This finding is comparable with the study finding of Israel (43 months) [21]. Nevertheless, it is lower when compared with the mean length of delay in the study conducted in Gondar (85.8 \pm 80.2 months) [12]. The most likely justification for this discrepancy is that in our study the patients with POP visited the hospital for seeking treatments by themselves. Meanwhile, in the Gondar study, there was a community-based screening and campaign for searching the cases of POP and, in this case, a lot of cases of POP may surveyed for the treatments and several cases with longer duration may contribute to the high value of estimates because in the 
Table 4 Reasons for delayed POP treatments among participants at selected General and Referral Hospitals of Southern Ethiopia, 2020

\begin{tabular}{|c|c|c|c|c|}
\hline Variables & $\begin{array}{l}\text { Delayed treatment } \\
(n=104)\end{array}$ & Early treatment $(n=19)$ & Total $(n=123)$ & Percent (\%) \\
\hline \multicolumn{5}{|c|}{ Lack of support } \\
\hline No & 38 & 13 & 51 & 41.46 \\
\hline Yes & 66 & 6 & 72 & 58.54 \\
\hline \multicolumn{5}{|c|}{ Low income } \\
\hline No & 49 & 15 & 64 & 52.03 \\
\hline Yes & 55 & 4 & 59 & 47.97 \\
\hline \multicolumn{5}{|c|}{ Lack of transportation } \\
\hline No & 19 & 14 & 33 & 26.83 \\
\hline Yes & 85 & 5 & 90 & 73.17 \\
\hline \multicolumn{5}{|c|}{ Unavailability of treatment centers } \\
\hline No & 75 & 16 & 91 & 78.98 \\
\hline Yes & 29 & 3 & 32 & 21.02 \\
\hline \multicolumn{5}{|c|}{ Believing POP as normal } \\
\hline No & 70 & 18 & 88 & 71.54 \\
\hline Yes & 34 & 1 & 35 & 28.46 \\
\hline \multicolumn{5}{|c|}{ Fear of surgery } \\
\hline No & 53 & 12 & 65 & 52.84 \\
\hline Yes & 51 & 7 & 58 & 47.16 \\
\hline \multicolumn{5}{|c|}{ Culture influence } \\
\hline No & 58 & 13 & 71 & 57.72 \\
\hline Yes & 46 & 6 & 52 & 42.18 \\
\hline \multicolumn{5}{|c|}{ Fear of stigma } \\
\hline No & 40 & 14 & 54 & 43.90 \\
\hline Yes & 64 & 5 & 69 & 46.10 \\
\hline \multicolumn{5}{|c|}{ Fear of disclosure } \\
\hline No & 42 & 14 & 56 & 45.53 \\
\hline Yes & 62 & 5 & 67 & 54.47 \\
\hline \multicolumn{5}{|l|}{ Being busy } \\
\hline No & 62 & 11 & 73 & 59.34 \\
\hline Yes & 42 & 8 & 50 & 40.66 \\
\hline
\end{tabular}

community we can find a woman who stayed for longer period by hiding the problem.

This study revealed women with a lack of support were over five times more likely to delay treatments of POP than their counterparts. This is inconsistent with the report from the study of Gondar saying lack of support is not associated with delaying for the treatments [12]. The possible justification for this discrepancy is most probably the POP care in Gondar is integrated into the fistula care center. In Gondar, women with POP are free of any payment related to POP treatments including transportation.

In this study, low income was significantly associated with delayed POP treatments. This is supported by the study conducted in Gondar, which reported the women with a lack of money were about two times more probably delay POP treatments $(\mathrm{AOR}=2,95 \% \mathrm{CI} 1.01,3.86)$ [12]. Women with a shortage of income were not able to afford the health service expenses, transport, food, and other costs $[22,23]$. It is a fact that most rural mothers in Ethiopia are not economically empowered [23]. Besides, in our context, women have many responsibilities that should carry out in day-to-day activities but not to generate income, and the power to make the financial decision is not by their hands and these and thus situation doubles their problems [23].

In this study, fear of social stigma increases the likelihood to delay treatments. A study conducted to assess reasons for delayed treatments of POP and fistula patients at the University of Gondar Comprehensive and 
Table 5 Logistic regression analysis about factors associated with the delay in seeking treatment of POP patients at selected general and referral Hospitals of Southern Ethiopia, 2020

\begin{tabular}{|c|c|c|c|c|}
\hline Variables & Delayed treatments & Early treatment & $\operatorname{COR}(95 \% \mathrm{Cl})$ & AOR $(95 \% \mathrm{CI})$ \\
\hline \multicolumn{5}{|c|}{ Lack of support } \\
\hline No & $38(74.5 \%)$ & $13(25.5 \%)$ & 1 & 1 \\
\hline Yes & $66(91.7 \%)$ & $6(8.3 \%)$ & $5.2(2.0-15.36)$ & $5.2(1.42-19.5)^{*}$ \\
\hline \multicolumn{5}{|c|}{ Low income } \\
\hline No & $49(76.6 \%)$ & $15(23.4 \%)$ & 1 & 1 \\
\hline Yes & $55(93.2 \%)$ & $4(6.8 \%)$ & $4.2(1.3-13.54)$ & $5.8(1.1-19.66)^{*}$ \\
\hline \multicolumn{5}{|c|}{ Lack of transportation } \\
\hline No & $19(57.6 \%)$ & $14(42.4 \%)$ & 1 & 1 \\
\hline Yes & 85 (94.4\%) & $5(5.6 \%)$ & $8(2.628-24.26)$ & $2.2(0.56-8.48)$ \\
\hline \multicolumn{5}{|c|}{ Unavailability of treatment centers } \\
\hline No & $75(82.45)$ & $16(17.6 \%)$ & 1 & \\
\hline Yes & $29(90.6 \%)$ & $3(9.4 \%)$ & $2.06(0.56-7.6)$ & - \\
\hline \multicolumn{5}{|c|}{ Believing POP as normal } \\
\hline No & $70(79.5 \%)$ & $18(20.5 \%)$ & 1 & 1 \\
\hline Yes & $34(97.1)$ & $1(2.9 \%)$ & $8.74(1.12-68.2)$ & $9.9(0.96-11.3)$ \\
\hline \multicolumn{5}{|c|}{ Fear of surgery } \\
\hline No & $53(81.5 \%)$ & $12(18.5 \%)$ & 1 & 1 \\
\hline Yes & $51(87.9 \%)$ & $7(12.1 \%)$ & $1.65(0.6-4.52)$ & - \\
\hline \multicolumn{5}{|c|}{ Culture influence } \\
\hline No & $58(81.7 \%)$ & $13(18.3 \%)$ & 1 & \\
\hline Yes & $46(88.5 \%)$ & $6(11.5 \%)$ & $1.7(0.61-4.87)$ & - \\
\hline \multicolumn{5}{|c|}{ Fear of social stigma } \\
\hline No & $40(74.1 \%)$ & $14(25.9 \%)$ & 1 & 1 \\
\hline Yes & $64(92.8)$ & $5(7.2 \%)$ & $4.48(1.5-13.39)$ & $4.7(1.2-18.59)^{*}$ \\
\hline \multicolumn{5}{|c|}{ Fear of disclosure } \\
\hline No & $42(75 \%)$ & $14(25 \%)$ & 1 & 1 \\
\hline Yes & $62(92.5 \%)$ & $5(7.5 \%)$ & $4.1(1.4-12.34)$ & $4(0.99-16.43)$ \\
\hline \multicolumn{5}{|l|}{ Being busy } \\
\hline No & $62(84.9 \%)$ & $11(15.1 \%)$ & 1 & \\
\hline Yes & $42(84 \%)$ & $8(16 \%)$ & $0.93(0.35-2.51)$ & - \\
\hline
\end{tabular}

Key: AOR, adjusted odds ratios; COR, crude odds ratios

* Statistically significant at $P$ value $\leq 0.05$ in multivariable logistic regression analysis

Specialized Hospital, Ethiopia, reported fearing social stigma was not associated with delay for treatments of POP. However, that study reported women who fear disclosing fistula owing to social stigma were two times more likely to delay the fistula treatments when compared with their counterparts [12]. The possible justification for the discrepancy is most probable Hamlin Fistula Center in Gondar has been delivering integrated service of fistula and POP care, repeatedly conducting the POP campaign and expanding awareness creation about the problem in the Gondar community.

In this study, out of 123 POP patients, $7.3 \%$ were divorced due to POP. This is comparable with the report of a study from Uganda that reported their husband rejected about $25 \%$ of women with POP [20]. The same was true in Nepal among women with POP [24] and in Gondar among women with fistula [12]. Moreover, qualitative studies in Nepal and Bahr Dar also believe that social stigma is the most important barrier to seek health care and to expose problems even to their close ones feeling it shameful because POP involves the most sensitive part of the body (genitalia) [16, 24]. Furthermore, due to fear of discrimination and rejection from people, they hide the illness at all and they disclose when it gets severe and unbearable after an extended duration of time since they begin to feel symptoms. Because of this delay, the problem reaches its advanced stage, which causes surgical interventions to become the only possible treatment solution. Notably, most women in Southern Ethiopia have less awareness about the POP and unable to reveal 
their problems related to their genitalia (sensitive body parts) because of the feeling of shamefulness, which has a deep root in the culture of the society.

As a limitation, this study was hospital-based and, hence, lacks generalizability to the community at large. Moreover, further study is needed in different places and the population.

\section{Conclusions}

In this study, most of the POP patients were delayed for POP treatments. Factors like lack of support, low income, and fear of losing social value/stigma were associated with the delay in seeking treatment. Screening for the POP cases, educating (making awareness) the community about this devastating disease to facilitate early treatment and to avoid social stigma, and raising access to treatment by making the nearby hospitals equipped with facilities to treat POP are recommended.

\section{Abbreviations}

AOR: Adjusted odds ratios; COR: Crude odds ratios; POP: Pelvic organ prolapse; SNNPR: South Nations Nationalities and Peoples' Region.

\section{Acknowledgements}

We would like to pass our deepest gratitude to the College of Medicine and Health Sciences, University of Gondar, for creating such a conducive learning environment, providing financial support, and giving this chance to conduct this project. Moreover, our thanks would be to the hospital directors, data collectors, and study participants for their commitment during data collection.

\section{Authors' contributions}

$A B, M O, Y A$, and $A W$ participated in conceptualization, formal analysis, investigation, methodology, supervision, visualization, writing-original draft, writing-review and editing, and approving the final draft. All authors read and approved the final manuscript.

\section{Funding}

This study was funded by the University of Gondar (http://UoG.edu.et) for AB. Sponsors or funders did not play role in study design, data collection and analysis.

\section{Availability of data and materials}

The datasets used and/or analyzed during the current study are available from the corresponding author on reasonable request.

\section{Declarations}

Ethical approval and consent to participate

Ethical clearance was obtained from the Ethical Review Committee of the College of Medicine and Health Sciences, University of Gondar. Permission letters were obtained from the SNNPR Health Bureau and the administrations of each hospital. After a brief explanation about the objectives of the study for the participants, written informed consent was obtained from study participants. Furthermore, confidentiality was kept carefully by using codes instead of any personal identifiers, and privacy was maintained given that the standard medical examination/practice has been done. We confirm that all methods were performed in accordance with the relevant guidelines and regulations.

\section{Consent for publication}

Not applicable.

\section{Competing interests}

The authors declare that they have no competing interests.

\section{Author details}

1 Department of Human Anatomy, College of Medicine and Health Sciences, Hawassa University, Hawassa, Ethiopia. ${ }^{2}$ Department of Human Anatomy, School of Medicine, College of Medicine and Health Sciences, University of Gondar, Gondar, Ethiopia. ${ }^{3}$ Department of Epidemiology, Institute of Public Health, College of Medicine and Health Sciences, University of Gondar, Gondar, Ethiopia.

Received: 17 October 2020 Accepted: 22 February 2021

Published online: 01 March 2021

\section{References}

1. Iglesia CB, Smithling KR. Pelvic organ prolapse. Am Fam Phys. 2017:96(3):179-85.

2. Sharma A, Zhang JP. Risk factors and symptoms of uterine prolapse: reality of Nepali women. Asian Women. 2014;30(1):81-95.

3. Belayneh T, Gebeyehu A, Adefris M, Rortveit G, Awoke T. Pelvic organ prolapse in Northwest Ethiopia: a population-based study. Int Urogynecol J. 2019;31:1873-81.

4. Horst W, Do Valle JB, Silva JC, Gascho CLL. Pelvic organ prolapse: prevalence and risk factors in a Brazilian population. Int Urogynecol J. 2017;28(8):1165-70.

5. Masenga GG, Shayo BC, Rasch V. Prevalence and risk factors for pelvic organ prolapse in Kilimanjaro, Tanzania: a population based study in Tanzanian rural community. PLoS ONE. 2018;13(4):e0195910.

6. Li Z, Xu T, Li Z, Gong J, Liu Q, Zhu L. An epidemiologic study of pelvic organ prolapse in rural Chinese women: a population-based sample in China. Int Urogynecol J. 2019;30(11):1925-32.

7. Dheresa M, Worku A, Oljira L, Mengistie B, Assefa N, Berhane Y. Factors associated with pelvic floor disorders in Kersa District, Eastern Ethiopia: a community-based study. Int Urogynecol J. 2019;30(9):1559-64.

8. Aytan H, Ertunç D, Tok EC, Yaşa O, Nazik H. Prevalence of pelvic organ prolapse and related factors in a general female population. Turk J Obstet Gynecol. 2014;11(3):176.

9. Gurjar B, Kedar K. Pelvic organ prolapse-a concern. J Evol Med Dent Sci. 2017;6(14):1080-3.

10. El Kady OSH, Tamara TF, El Mohsen Sabaa HA, Shawky Hafez AHM. Assessment of the prevalence of pelvic floor disorders in both vaginal and cesarean deliveries and their impact on the quality of life. Egypt $J$ Hosp Med. 2017;68(2):1252-6.

11. Eleje G, Udegbunam O, Ofojebe C, Adichie C. Determinants and management outcomes of pelvic organ prolapse in a low resource setting. Ann Med Health Sci Res. 2014;4(5):796-801.

12. Adefris M, Abebe SM, Terefe K, Gelagay AA, Adigo A, Amare S, et al. Reasons for delay in decision making and reaching health facility among obstetric fistula and pelvic organ prolapse patients in Gondar University hospital, Northwest Ethiopia. BMC Womens Health. 2017;17(1):64.

13. Akmel M, Segni H. Pelvic organ prolapse in Jimma University Specialized Hospital, Southwest Ethiopia. Ethiop J Health Sci. 2012;22(2):85-92.

14. Hurissa T, Bekele D. A one-year review of pelvic organ prolapse at St. Paul's Hospital Millennium Medical College, Addis Ababa Ethiopia. 2018.

15. Henok A. Prevalence and factors associated with pelvic organ prolapse among pedestrian back-loading women in Bench Maji Zone. Ethiop J Health Sci. 2017;27(3):263-72.

16. Gjerde JL, Rortveit $G$, Muleta M, Adefris M, Blystad A. Living with pelvic organ prolapse: voices of women from Amhara region, Ethiopia. Int Urogynecol J. 2017;28(3):361-6.

17. Chen G-D, Ng S-C. Updated definition of female pelvic organ prolapse. Incont Pelvic Floor Dysfunct. 2007;1 (4):121-4.

18. Schreiner M. Estimating consumption-based poverty in the Ethiopia Demographic and Health Survey. Ethiop J Econ. 2012;21(1):73-106.

19. Adelowo A, O'Neal E, Hota L. Underlying factors contributing to the delay in patients seeking care for pelvic floor dysfunction. Clin Trials J. 2014;4(174):2167.

20. Krause HG, Natukunda H, Singasi I, Hicks SS, Goh JT. Treatment-seeking behaviour and social status of women with pelvic organ prolapse, 4th-degree obstetric tears, and obstetric fistula in western Uganda. Int Urogynecol J. 2014;25(11):1555-9. 
21. Krissi H, Eitan R, Edward R, Peled Y. Diagnostic delay in secondary care for lower urinary tract and pelvic organ prolapse symptoms in women. Arch Gynecol Obstet. 2012;286(1):115-8.

22. Baker Z, Bellows B, Bach R, Warren C. Barriers to obstetric fistula treatment in low-income countries: a systematic review. Tro Med Int Health. 2017;22(8):938-59.

23. AAE CSA: Ethiopian Demographic Health Survey. In: ICF international. 2011.

24. Shrestha B, Onta S, Choulagai B, Poudyal A, Pahari DP, Uprety A, et al. Women's experiences and health care-seeking practices in relation to uterine prolapse in a hill district of Nepal. BMC Womens Health. 2014;14(1):20

\section{Publisher's Note}

Springer Nature remains neutral with regard to jurisdictional claims in published maps and institutional affiliations.
Ready to submit your research? Choose BMC and benefit from:

- fast, convenient online submission

- thorough peer review by experienced researchers in your field

- rapid publication on acceptance

- support for research data, including large and complex data types

- gold Open Access which fosters wider collaboration and increased citations

- maximum visibility for your research: over $100 \mathrm{M}$ website views per year

At BMC, research is always in progress.

Learn more biomedcentral.com/submissions 